\title{
EQ-5D and the EuroQol Group: Past, Present and Future
}

\author{
Nancy J. Devlin ${ }^{1} \cdot$ Richard Brooks ${ }^{2}$
}

Published online: 13 February 2017

(C) The Author(s) 2017. This article is published with open access at Springerlink.com

\begin{abstract}
Over the period 1987-1991 an inter-disciplinary five-country group developed the EuroQol instrument, a five-dimensional three-level generic measure subsequently termed the 'EQ-5D'. It was designed to measure and value health status. The salient features of its development and its consolidation and expansion are discussed. Initial expansion came, in particular, in the form of new language versions. Their development raised translation and semantic issues, experience with which helped feed into the design of two further instruments, the EQ-5D-5L and the youth version EQ-5D-Y. The expanded usage across clinical programmes, disease and condition areas, population surveys, patient-reported outcomes, and value sets is outlined. Valuation has been of continued relevance for the Group as this has allowed its instruments to be utilised as part of the economic appraisal of health programmes and their incorporation into health technology assessments. The future of the Group is considered in the context of: (1) its scientific strategy, (2) changes in the external environment affecting the demand for EQ-5D, and (3) a variety of issues it is facing in the context of the design of the instrument, its use in health technology assessment, and potential new uses for EQ-5D outside of clinical trials and technology appraisal.
\end{abstract}

Electronic supplementary material The online version of this article (doi:10.1007/s40258-017-0310-5) contains supplementary material, which is available to authorized users.

Nancy J. Devlin

ndevlin@ohe.org

1 Office of Health Economics, London SW1 3QT, UK

2 EuroQol Group, Rotterdam, The Netherlands

\section{Key Points for Decision Makers}

The EQ-5D portfolio of health status instruments, EQ-5D-3L, EQ-5D-5L and EQ-5D-Y, is the product of nearly 30 years of development and adaptation.

These instruments have been applied in a variety of health sector settings, in patient-reported outcome exercises, in population health studies, and in health technology assessment.

A wide-ranging programme of research studies is in process to adapt and further develop the EuroQol portfolio.

\section{Introduction}

The EQ-5D is a well-known and widely used health status instrument. It was developed by the EuroQol Group in the 1980s to provide a concise, generic instrument that could be used to measure, compare and value health status across disease areas.

The initial Group participants were from a variety of professional backgrounds with a common interest in the measurement of health status and in the outcomes of healthcare programmes. The development of the instrument was motivated in part by health economics considerations, i.e. to create a way of measuring health status to inform resource allocation decisions by enabling the application of cost-effectiveness analysis (CEA) to health care. Aiming at valuing health states gave the potential for the instrument to estimate quality-adjusted life-years (QALYs) for use in CEA. 
Valuation, however, was not the sole consideration of the Group, and this has been confirmed by the incorporation of EQ-5D into clinical trials, observational studies, population health surveys and, more recently, into routine outcome measurement via Patient Reported Outcome (PRO) measures in the healthcare sector.

The aims of this paper are to: (1) describe the provenance and development of the EQ-5D by the EuroQol Group, and to highlight the factors that contributed to its widespread use as a generic instrument; (2) outline the current state of play with respect to the use and application of EQ-5D and further development of the suite of EQ-5D instruments, and (3) consider the future of the EQ-5D, including the research directions signalled by the Group, and the challenges that might shape its future use and development.

\section{A Brief History of the EQ-5D}

Detailed histories of the EuroQol Group and its development of the EQ-5D are available elsewhere [1-3]. In this paper the focus is on the following questions: Why was the EQ-5D initially developed? How did its use and application evolve? How will the EuroQol Group proceed?

\subsection{The Provenance of the EQ-5D}

The EuroQol Group started its journey in 1987: 14 people met to exchange ideas about how to approach the development of a health status measurement instrument. One of the motivations for doing so was to assist healthcare decision-makers to make resource allocation decisions informed by evidence on the cost-effectiveness of alternative treatments.

From the outset the Group therefore sought a 'common core' of basic information or key attributes to be collected by all investigators in a standardised way. This came to be crystallised in the following set of objectives: (1) To develop a generic instrument to describe and value healthrelated quality of life (HRQoL), providing both a descriptive profile and an overall index. (2) It was to be a standardised tool to facilitate the collection and pooling of a common data set. (3) It was to be suitable for selfcompletion and acceptable for use in postal surveys (at that point, a common mode of data collection). These objectives in turn led to a number of requirements for the descriptive system: (1) dimensions should be relevant to patients across the spectrum of health care and to members of the general population. (2) It should be simple-using as few dimensions as possible, with as few levels as possible within each dimension. (3) It should be amenable to selfcompletion in a range of settings, should be simple enough not to require detailed instructions, and should only take a couple of minutes to complete.

The Group discussed various alternatives with respect to the selection of dimensions, including a survey of patients and the general population, to identify common dimensions of relevance to all groups. Since the selection of dimensions from such an exercise would still involve value judgments, the Group members decided instead to draw on their own expertise by undertaking a detailed review of other available generic health measures. Contrary to expectations, the dimensions suggested for inclusion as a result of this exercise were broadly similar, differing more on dimension nomenclature than on content. General agreement settled on the following: mobility, daily activities and self-care, psychological functioning, social and role performance, and pain or other health problems [4]. In addition, as the Group was multilingual, the classification system descriptors were selected from the outset with a number of languages in mind, rather than a source version being translated into other languages.

The EuroQol Group publicly introduced a six-dimensional health status instrument after some 3 years of development [1]. However, by the time of its publication, further empirical testing had already led the instrument to be further refined to five dimensions-mobility, self-care, usual activities, pain/discomfort and anxiety/depression-each with three levels. Originally named the 'EuroQol instrument,' it was formally designated 'EQ-5D' in 1995. The descriptive system defined $\left(3^{5}\right)=243$ different states. Two further states were initially included in valuation work: unconscious and dead (both states undefinable in terms of the descriptive system). With the development of the five-level version EQ-5D-5L (see Sect. 2.4), the three-level version was re-designated EQ-3D-3L. (Both versions appear as Appendices 1-4 in the online Supplementary Material).

Initial EQ-5D valuation work employed ranking, magnitude estimation and visual analogue scale (VAS) approaches, but VAS was quickly established to be the valuation approach of choice. At that point in time, other methods were in their infancy, such as time trade-off (TTO), or had not been much applied in the health status context, such as the standard gamble (SG). It was for that reason that the EQ VAS was introduced as part of the EQ5D questionnaire right from the start: its initial role was actually as a warm-up task for the VAS valuation tasks, and only later was its potential usefulness as a self-reported global measure of overall health recognised [5].

While these early efforts converged on a descriptive system in what was a relatively short period of time, a considerable and rapidly expanding research programme continued, to test the reliability and validity of the EQ-5D in populations and patients. This was accompanied by an extensive programme of research on the valuation of EQ- 
$5 \mathrm{D}$, to test the effect on values of the stated duration of states; the visual presentation and positioning on the VAS scale; the selection of the states to be valued; and deliberation about whose values (experts, patients, or the general public) should be used. It is important to note that these efforts preceded - by over a decade - the establishment of formal health technology assessment (HTA) organisations and processes, so the EuroQol Group was operating in largely uncharted territory.

\subsection{Consolidation and Expansion}

The two and a half decades which followed the establishment of the EQ-5D in 1990 were characterised by continued research and development, considerable growth in the use of the EQ-5D in healthcare decision-making, and ongoing efforts to develop both additional instruments within the EQ-5D framework and improved methodologies for eliciting and modelling health state values. There were also significant changes in the EuroQol Group as an organisation-it grew, formalised its processes and put in place the business model which exists today.

First, it is noteworthy that, apart from some minor wording and design changes to the original EQ-5D questionnaire, what is now termed EQ-5D-3L has remained more or less unchanged from 1990 to the present day. While there has been ongoing experimentation with additional dimensions and the number of levels, as discussed below, these changes have not been incorporated in the EQ-5D-3L instrument itself.

This stability in the EQ-5D 3L instrument has had a number of consequences. After two and a half decades of use and research, there is a substantial back-catalogue of studies, evidence and EQ-5D data available to support new investigations. Research has built upon and developed knowledge of the use and analysis of EQ-5D data. From the perspective of its application in HTA, this stability can facilitate consistent decisions over time.

Expansion in the use of EQ-5D post-1990 came in a number of ways. First, the demand for EQ-5D data and the accompanying value sets increased markedly as HTA organisations became established in healthcare systems around the world. Second, considerable resources were devoted to expanding the number of EQ-5D language versions, facilitating global use of the instrument. Third, there was a rapid increase in the number of applications for licences to use the EQ-5D in a variety of medical and health sector settings, and pharmaceutical companies began to use the instrument in increasing numbers, reflecting the requirement of HTA bodies to supply evidence on QALYs.

In the valuation context a noteworthy development came from the Measurement and Valuation of Health (MVH) study, led from the University of York in the early 1990s, in the form of a set of EQ-5D 'tariffs' based on TTO values from the general public which could be used to generate QALYs. The tariff (value set) produced from the MVH study [6] became very widely applied in economic evaluation, both in the UK and in other countries (and continues to be used today). This subsequently led to a number of other countries adopting similar methods for collecting and modelling their own value sets.

Also of significance for the progress of the EuroQol Group was the EQ-net project of 1998-2001 funded by the Biomed programme of the European Commission. This project provided the opportunity to put members' research work into a more structured context, with most of the efforts of the Group devoted to it during this 3-year period.

The tasks involved were divided into three sub-projects: Translation, Valuation and Application. In addition, the communication of information and knowledge about EQ$5 \mathrm{D}$ was addressed, with detail on all aspects of the project subsequently being published in book form [7]. Since the main aim of the project was to harmonise data on the valuation of EQ-5D health states collected in different European countries, considerable effort was put into the Valuation sub-project. Two databases were established, one containing VAS valuations and the other TTO valuations. The Application sub-project produced standard operating procedures (SOPs) for the design, analysis and reporting of EQ-5D in clinical, economic and population studies, which were included in the book alongside guidelines for differing modes of administration of EQ-5D: versions for observer, face-to-face administration, proxy and telephone. The work accomplished in the Translation sub-project is treated separately in Sect. 2.4 below.

Essentially the EQ-net project stimulated the further development and dissemination of EQ-5D, which fed into the scientific programmes pursued in the new millennium.

\subsection{The Evolution of the EuroQol Group as an Organisation}

As use of the instrument grew, the relatively simple clublike nature of the early Group necessarily evolved into more formalised arrangements. The use of EQ-5D in HTA (particularly by NICE in the UK, which, in 2004, identified the EQ-5D as its preferred instrument [8]) led to increased demand from pharmaceutical companies wanting to include EQ-5D data in HTA submissions. This presented an opportunity to license that use and to generate revenue. A key period in the evolution of the Group in this respect was 1993/94. Up until 1993, the activities of the Group were supported exclusively by the initial small group of members and their institutions, both by contributing their time and, occasionally, by contributing financial support to the enterprise. 
The next development was the appointment of a Business Manager and a Management Assistant in 1993 and 1994, respectively. In 1994, inquiries from the pharmaceutical industry began to be directed through the business office, which was instructed to develop a pricing policy. This marked the beginning of modest revenue generation, in keeping with the not-for-profit nature of the Group.

This was quickly followed by setting in place legal arrangements. In 1995 the formal organisational (and legal) structure for the Group comprising the EuroQol Association and Foundation, monitored by a Board and Executive Committee, were established under Dutch law, and a Business Management office set up in Rotterdam.

Critical to understanding the current nature of the EuroQol Group was the business model which emerged from this process of formalising the organisation in the mid-1990s. The key features are:

- The EuroQol Group in all its activities is a not-forprofit organisation.

- Users must register use of the instrument (copyright to which was first asserted in 1990 and was formally transferred to the Association upon its establishment in 1995).

- Commercial, for-profit users are changed a licence fee for the use of EQ-5D.

- Not-for-profit academic users are able to use EQ-5D free of charge.

- The EuroQol Group comprises both a business unit and an international, multi-disciplinary collaborative network of researchers - the members of the EuroQol Group - who drive forward the science surrounding the EQ-5D.

This combination of arrangements proved an appropriate model for promulgating the use of EQ-5D and generated revenue with which to support and fund research. Group membership expanded-currently at around 80and has become an international rather than a European network. In addition, with some members having a careerlong association with the Group there has been a great deal of continuity of endeavour.

\subsection{Instrument Development}

In Sect. 2.2 we noted that the EQ-5D-3L as an instrument has remained largely unchanged from 1990 until the present. However, there have been important related developments, including many new language versions, newly derived EuroQol Group instruments and systematic approaches to valuation for use in producing value sets. We briefly review the principal developments below, after outlining translation and version management issues.

\subsubsection{Translation and Version Management}

From the outset English had been used as the working or 'source' language for the EQ-5D, and the instrument was simultaneously constructed in Finnish, Dutch, Swedish and Norwegian. Draft translation guidelines were first developed in 1994, and in 1996 expanded guidelines were implemented, overseen by a Translations Committee, setting in place a standard forwards-and-backwards translations process which supported the development of a large number of language versions in subsequent years.

When EQ-5D spread to new languages the process of translation pointed to difficulties in language usage and to differences in the conceptualisation of EQ-5D dimensions and items across countries and languages. This led the Group to consider more closely the meanings of concepts and the related wording used in EQ-5D, not least in English. The Translation aspect of the EQ-net project provided the opportunity for substantive work on these matters. A definition of EQ-5D concepts was provided and a series of recommendations for further research was made [9]. Also generated were a taxonomy of definitions of EQ-5D concepts, SOPs and detailed translation guidelines. A detailed account of translating EQ-5D into 11 European languages provided an insight into the translation process, and the challenges involved [10].

In 2009 a Version Management Group (subsequently Committee) was established, with responsibilities for reviewing new language versions, responding to client and translation agency queries, updating essential documentation, and implementing systems aimed at improving version control and management. This group has responsibility not just for different language versions, but also for testing and approving electronic versions of the EQ-5D (tablet, web-based, PDAs), demand for which has risen [11].

\subsubsection{EQ-5D-5L}

Notwithstanding the strong uptake in the use of EQ-5D, particularly in HTA, concerns about its adequacy as a measure of HRQoL have been voiced. There continued to be lively debate within the EuroQol Group going back to 1994 [12] regarding the three-level structure of the response options (no, some, extreme problems/unable to) and whether this was associated with ceiling effects and a lack of sensitivity to changes in health. Kind and Macran fuelled that debate, reporting an investigation of a fivelevel version of the core five dimensions [13]. In 2005, sufficient momentum on this issue had built such that a EuroQol Group task force was established to consider an increased level descriptive system, in response to concerns 
about the perceived lack of sensitivity of the EQ-5D and ceiling effects in the descriptive system.

In 2006, after considerable debate and pre-studies about whether to go for a four- or five-level version, it was decided to recommend the development of a five-level version of the instrument, while retaining the same core five dimensions. In addition 'confined to bed' was replaced by 'unable to walk about' to increase sensitivity of the mobility dimension. Results from initial studies testing five-level versions of the EQ-5D showed increased reliability, sensitivity (discriminatory power) and feasibility [14-17].

As with the original EQ-5D, the intention behind the new five-level version was that it be accompanied by value sets. For that reason, rather than assign the additional two levels as 'unlabelled' response options between no and some, and some and extreme, problems, it was felt that all five levels required labels. Labels were selected following the results of semantic testing in England, Spain and France [18, 19], and the resulting labels translated into other languages. Two features of this process can be highlighted. First, the labels were chosen for UK English, Spanish and French, based on an exhaustive process of response scaling among a wide range of potential labels selected from the literature and existing PRO questionnaires, together with follow-up focus group research to explore respondents' understanding of those labels in the three countries. Second, once the labels had been decided on, the UK English, Spanish and French versions could then be used as source content for any new language versions required. These are produced following the EuroQol Group's translation methodology, which also includes in-depth semantic testing of all wording in the target language, with a particular focus on the severity labels (see [11]).

The new instrument was approved as an official EuroQol instrument in 2009. From that point, the five-level instrument has been referred to as the EQ-5D-5L, and the original EQ-5D has been re-named the EQ-5D-3L. The research underpinning the EQ-5D-5L is summarised in Herdman et al. [20], including consideration of the mapping of health states from one system to the other.

\subsubsection{EQ-5D-Y}

The EQ-5D was, implicitly, designed for self-completion by adults. However, HTA bodies and other healthcare decision-makers frequently make decisions regarding treatments for children and young people. From 1998, interest grew in the possibility of using the EQ-5D, or adapting it in some way, for use in younger people. An initial 'child friendly' version of the EQ-5D, reported in 2002 [21], was followed by research efforts in a range of countries. A task force, established in 2006, coordinated these efforts, and considered issues regarding what dimensions to include and how to label them, what number of levels to use, proxy completion, what age ranges to target, and how to value children's health states [22, 23].

These efforts culminated, in 2009, in approval of a 'youth' version, the EQ-5D-Y, as an official EQ-5D product. The EQ-5D-Y retained the same five-dimension, three-level format of the EQ-5D, but dimensions were described in more appropriate language as: mobility (walking about); looking after myself; doing usual activities; having pain or discomfort; feeling worried, sad or unhappy. (EQ-5D-Y appears as Appendix 5 in the online Supplementary Material).

EQ-5D-Y is suitable for self-completion by children aged 8-11 years; it is also recommended for use at ages 12-15 years, although use of the EQ-5D adult version might be possible in some circumstances. The EQ-5D adult version is recommended for those aged 16 years and over.

\subsubsection{Protocols for Value Sets}

Despite the widespread utilisation of the MVH tariff for QALY purposes there was no 'official' valuation protocol or consensus view within the EuroQol Group about valuation methods to be used in producing value sets. Different research teams adapted the MVH study design in various ways, making different choices about, for example, the number and selection of states to value; 'exclusion rules' applied to the data; and so on [24]. This limited the comparability of the data. This was addressed in 2009 at a meeting in Paris, where a modified version of the MVH study design was endorsed ('the Paris protocol') for use in EQ-5D-3L value set studies. Included among the changes incorporated at that point was dropping 'unconscious' from the states to be valued in such studies.

Having developed the EQ-5D-5L, the EuroQol Group decided this presented an important opportunity to improve valuation methods and to promote a consistent approach to valuing EQ-5D-5L by providing an official protocol and study design. Interim values for the EQ-5D-5L were available from a 'crosswalk' study: six countries administered both the five-level and three-level versions in parallel, from which a crosswalk enabled EQ-5D-5L profiles to be mapped to EQ-5D-3L profiles, and values applied from existing EQ-5D-3L value sets [25]. At the same time, a series of methodological studies were undertaken, exploring a variety of approaches to both TTO and discrete choice experiments (DCE). The latter method had been investigated in 2008 using the three-level version [26]. Work was also undertaken to develop thesoftware to allow these methods to be implemented in computer-assisted personal interviews (CAPI). A prototype protocol, incorporating these approaches, was piloted in a multi-country study [27].

Following further testing and refinement, the international protocol for valuation of EQ-5D-5L was launched 
[28]. This comprises a fully documented study design, interviewer guide, interviewer training materials and the CAPI software, EQ-VT (EuroQol Valuation Technology). Valuation studies in England, The Netherlands, Spain and Canada commenced in 2012, coordinated and supported by the EuroQol Executive Office.

\section{EQ-5D: The Current State of Play}

The EuroQol Group operates within a formal legal and organisational framework, and undertakes formal processes for decision-making to ensure accountability and transparency. The Executive Office in Rotterdam administers licensing arrangements, manages Group operations, provides scientific advice and undertakes research, and acts to support and coordinate the activities of all its members.

Its stated vision is:

"To improve decisions about health and health care throughout the world by developing, promoting and supporting the use of instruments with the widest possible applicability for the measurement and valuation of health" [29].

Its stated mission [30] is: (1) to provide leadership in the research and development of instruments that describe and value health. (2) To promote the use of instruments developed by the EuroQol Group and to support individuals and organizations across the world seeking to use those instruments. (3) To foster and support an international community of researchers whose activity informs the development and application of EuroQol Group instruments. (4) To ensure access to the accumulated research expertise of the EuroQol Group and to actively promote the transfer of knowledge and evidence regarding the use, analysis and interpretation of measures developed by the EuroQol Group. (5) To support early career researchers in the field of health and quality-of-life research through involvement in EuroQol Group activities.

\subsection{Availability and Use of EQ-5D Instruments}

The position in 2016 with respect to translations, as detailed on the EuroQol website (http://www.euroqol.org), is $176 \mathrm{EQ}-5 \mathrm{D}-3 \mathrm{~L}$ and $138 \mathrm{EQ}-5 \mathrm{D}-5 \mathrm{~L}$ language versions. In addition alternative available formats comprise: proxy, telephone interview, IVR (interactive voice response) via telephone, web, tablet and PDA.

The number of studies using the EQ-5D suite of instruments, registered with the EuroQol Group, totalled over 17,000 by 2015 . These comprised over 80 clinical areas and 40 programmes and settings. For studies in the top 25 clinical areas, apportioned by type of study, the figures were: interventional studies (including randomised control trials) $33 \%$, observational studies $28 \%$, surveys $15 \%$ and other studies $24 \%$. Programmes and settings included, inter alia, surgical procedures, general practice and primary care, hospital waiting lists, physiotherapy and rehabilitation.

Further information is shown in Tables 1 and 2.

\subsection{New and Emerging Uses of EQ-5D}

EQ-5D instruments continue to be used to measure the health status of patients, and to provide evidence on the cost-effectiveness of healthcare technologies, and in population health surveys to examine the health of the general public.

More recently, there has also been growing use of the EQ-5D as part of routine, administrative data collection in

Table 1 The EuroQol Group's current suite of instruments

\begin{tabular}{llll}
\hline & EQ-5D-3L & EQ-5D-5L & EQ-5D-Y \\
\hline Number of language (paper) versions available & 176 & 138 & 40 \\
Number of new requests to use the instrument in 2015 & 1416 & 2274 & 34 \\
Number of value sets available (+planned) & 25 & 22 & None: pilot projects currently underway \\
Number of observations collected in 2015 & $>1.3$ million & $>2.9$ million & 5600 \\
\hline
\end{tabular}

Source: EuroQol Executive Office

Table 2 Availability and use of EuroQol Group Valuation Technology (EQ-VT)

\section{EQ-VT}

Number of translated versions available

Value sets available or planned using EQ-VT $n=16$
22

Value sets available: Argentina, Canada, China, England, Hong Kong, Japan, Netherlands, Spain Value sets currently underway/planned: Germany, Indonesia, Ireland, Norway, Singapore, South Korea, Thailand, UK

Source: EuroQol Executive Office 
healthcare systems. In 2009, NHS England introduced its Patient Reported Outcome Measures (PROMs) programme: the EQ-5D, alongside condition-specific PROs, is collected from all NHS patients before and after elective surgery for hip and knee replacement, varicose veins and hernia repair [31]. These data are used to monitor the performance of healthcare providers, to incentivise quality by linking reimbursement to performance, and to inform patient choice of provider. Similar uses of EQ-5D are underway, or planned, in the healthcare system in Sweden, and in Alberta Health Services in Canada. The private healthcare sector also uses EQ-5D-for example, Southern Cross, New Zealand's largest private insurance company, require providers to collect these data as a means of checking the quality of care they fund. These PROMs programmes generate large-scale data-they cover entire populations, rather than samples, of patients, and are generating powerful real-world insights on treatment and provider effectiveness, beyond the confines of clinical trials. EQ-5D has thus become part of the 'big data' revolution in health care [32]. This emerging use poses new challenges for issues such as data sharing and methods to achieve comparability of responses.

\section{The Future of EQ-5D}

The future of the EQ-5D instruments will depend both on internal factors-the aims and scientific strategy of the Group-as well as on changes in the external environment, for example, developments in HTA, and new and emerging uses of EQ-5D outside HTA.

\subsection{The Scientific Strategy of the EuroQol Group}

In 2014 the EuroQol Group initiated a process to define its scientific strategy. The outcome included a revised and augmented system of six working groups. Each is tasked to drive forward research in a key area. This framework indicates the priorities of the EuroQol Group, and where future developments will take place as a result of these efforts. Table 3 lists these groups, and their major objectives.

Table 3 EuroQol working groups and their main objectives

Descriptive systems

To explore the conceptual basis for generic preference-based HRQL measures (i.e., descriptive and measurement work)

To investigate the conceptual basis and develop a framework to rationalize the development of various bolt-ons and bolt-offs

Valuation

To investigate new approaches to valuing health (including approaches within scope of the QALY framework)

To support the development and dissemination of EQ-5D-5L value sets in key countries and stimulate interest in producing EQ-5D (3L and

$5 \mathrm{~L})$ value sets in key countries

To support methodological research examining the basis/rationale for value sets for patient groups

To provide scientific guidance and support for existing protocols related to valuation studies

To provide scientific guidance and support for valuation of bolt-on studies

Large-scale applications

To promote and examine large-scale health systems applications for EQ products

To stimulate methodological and applied research relating to the use of EQ-5D in measuring local and system-wide performance, as well as its use in assessing population health

To focus on applications inside healthcare systems, as well as in other non-health sectors

EQ-5D in younger populations

To develop EQ-5D instruments suitable for use in younger populations of various age ranges

To promote research to explore the validity of the EQ-5D-Y as a measure of health status in younger populations

To work closely with the Working Group on Valuation in developing a work programme for the valuation of EQ-5D-Y states

To promote research in the field of application studies

To develop and to test possible bolt-ons and bolt-offs (with regard to the self-care dimension) for the EQ-5D-Y

Interface development

To support different end-users of the EQ-5D instruments who wish to use electronic or internet-based methods of data collection

Education and outreach

Organize regular educational meetings for interested EuroQol members on specific topics related to research and application of EQ-5D

Organize and promote regional meetings of researchers, decision-makers and users interested in EQ-5D outside Europe and North America Propose educational and uptake initiatives that could be supported by the EuroQol Foundation

Source: EuroQol Executive Office 
One aspect of these priorities is the intention to revisit the descriptive system. Systematic reviews of the use of EQ-5D indicate its acceptability as a measure of health status in most disease areas [33], although there are some types of health problems where the EQ-5D appears not to perform well, such as problems with hearing and vision.

Nevertheless, there have been considerable advances in health status measurement and the science of PRO instrument development since the initial development of EQ-5D in the late 1980s, hence the Group considered that it would be appropriate at this juncture to explore whether the instrument could be improved and its performance enhanced and, if so, in what ways. The Group has therefore signalled its intention to undertake a 'fundamental' research programme to address the conceptual and empirical basis for the core instrument, as indicated in Table 3. If evidence provides clear support for making changes to the conceptual model or to instrument content, this will lead to discussion around whether a new version of the instrument should be developed and the methods for doing so. This would clearly be a long-term research endeavour and an important part of the discussion would be the implications of making any significant changes.

The Group is also undertaking experiments on another approach to enhancing instrument performance in certain population sub-groups, i.e. through the use of 'bolt-on' dimensions. In its simplest form, this retains the core five dimensions, but adds one (or more) dimension to capture aspects of health which may not adequately captured by these dimensions. Some initial experiments with bolt-ons have been undertaken for vision, hearing, tiredness, psoriasis and sleep [34-36]. At a descriptive level, the introduction of bolt-on dimensions can add to the richness of respondent profile data. However, this approach raises nontrivial issues, especially in connection with the consequences for health state valuations in the expanded instruments $[33,36]$.

\subsection{Changes in the External Environment Affecting the Demand for EQ-5D}

The future of the EQ-5D is closely linked to developments in healthcare systems with respect to measuring outcomes, and how outcomes data are used by decision-makers. EQ5D has been a cornerstone of QALY-based HTA systems and its continued use in that context depends on the extent to which QALYs and cost-effectiveness analysis remain an integral part of HTA. Critiques of QALYs have long existed and continue to generate debate. In recent years, there has been growing interest in the inclusion of other elements of value that might be missed by QALYs, such as, inter alia, patients' preferences regarding their treatment, benefits to caregivers and social benefits from increased productivity. It has also been suggested that there needs to be a shift to new measurement paradigms-such as assessing treatment benefits in terms of gains or losses in subjective wellbeing. If such suggestions gain traction EQ5D may become less important in this context [37-40].

Notwithstanding the growing interest in going 'beyond QALYs', it seems likely that the QALY will remain a central part of HTA for some time to come-in part because of the challenges in implementing a radically different approach and the difficulty in making such a fundamental change, and in part because of the lack of any other credible measurement approach to 'value' which could replace it.

Furthermore, HTA systems are being established in ever more parts of the world-South America, Africa, Asia-so demand for EQ-5D instruments seems likely to continue to grow as these areas of the world require local evidence to inform their decisions.

EQ-5D usage expanded because the instrument provided a simple and effective means to compare the impact of disease and its treatment across different disease areas and patient groups, and of incorporating societal preferences for health states into those comparisons. A further factor which may affect future demand for EQ-5D is the rise of condition-specific instruments accompanied by utilities: this means that utility becomes the common denominator for comparison of QALYs (providing utilities are generated using consistent methods, and give commensurate measures of utility), not the generic health state descriptive system itself.

Finally, demand for EQ-5D will also be affected by its growing use outside of HTA, to inform different kinds of decisions. This includes the potential for EQ-5D to have a role in clinical settings, as part of patient decision aids [41] and the growing inclusion of EQ-5D in routine, administrative data in healthcare delivery, as reported in Sect. 3.2.

These new uses of EQ-5D may have implications for the future development of methodologies to support its use. For example, where EQ-5D is used to monitor the performance of providers, appropriate case-mix adjustment methods become crucial, as does an understanding of how much variation in performance is 'normal'. Given the large scale of health system-wide data collection of EQ-5D data in PROMs programmes, a better understanding of what constitutes a minimally important difference in EQ-5D arguably also becomes more important.

Further, these uses of EQ-5D serve quite different types of healthcare decisions and decision-makers (e.g. patients' decisions about what treatments to choose, regulators' monitoring of quality standards of healthcare providers) than those that have conventionally been the focus of EQ5D (e.g. budget holders' decisions about how resources are allocated in the healthcare sector or HTA decisions about 
whether new technologies are good value for money). This potentially has implications for the way EQ-5D profile data are summarised: the use of 'value sets' reflecting the preferences of the general public has a normative justification specific to the use of those data in resource allocation decisions [42]. In contrast, judgements about what constitutes 'good quality health care', or individual patients' choices between different treatments, might suggest a role for patient preferences, rather than those of the general public, in summarising and analysing EQ-5D data [43]. These are areas for future exploration.

\section{Discussion}

Four major factors in the development over time of EQ-5D can be discerned.

1. The determination from the outset that a generic health status instrument should be as short and simple as possible to minimise the burden of both measuring and valuing health status led first to its adoption by medical personnel, and then later supported and facilitated the burgeoning interest in economic evaluation in health care and the establishment of HTA by NICE and similar bodies internationally. Associated with these developments was the requirement laid on pharmaceutical companies to demonstrate the cost-effectiveness of their products. Initially, and for several years, the EuroQol Group maintained that its instrument was to be used 'alongside' other instruments in evaluating medical programmes, and indeed this has often been the case. However, EQ-5D increasingly came to be employed as a stand-alone instrument.

2. The 'not for profit' business model employed by the Group generated a stream of revenue from commercial users with which to fuel further research-while simultaneously allowing free use of the instrument by academics. This flow of funds has continued, and indeed increased in volume and value, thus enabling new and innovative research to be pursued by a Group whose membership and geographical scope has steadily expanded.

3. Another key aspect of the expansion in the utilisation of EQ-5D, geographically and otherwise, has been the 'open access' policy pursued by the Group from the outset. This included: publishing the proceedings of Plenary meetings, which are all available in both book form and on the EuroQol website; a commitment to publishing research in peer-reviewed journals wherever possible; and extended to an open access policy for Group publications.
4. Notwithstanding considerable experimental research over the last 25 years, the EQ-5D-3L instrument has remained fundamentally unchanged from its establishment in the early 1990s. New versions of the instrument have thus far retained the same five dimensions, but, in the case of EQ-5D-5L, expanded the number of levels, and in the case of EQ-5D-Y, adjusted the way dimensions are communicated. The overall outcome of this is that the EQ-5D is backed by over a quarter century of data, evidence, publications, and researcher and user experience. This offers considerable appeal to both researchers and decisionmakers: new data can be compared to EQ-5D population norms, EQ-5D evidence from specific disease groups, and so on.

There are, however, challenges in turning science into 'products'. EQ-5D-3L was developed with the clear intention of producing a generic instrument accompanied by values. EQ-5D-5L and EQ-5D-Y had the same objectives-but a notable difference in the development process underpinning these new instruments is that piloting of the valuation of health states has not fed into the instrument development phase in either case, but tended to take place after the instruments have been finalised. This has given rise to some issues. For example, difficulties have arisen for members of the general public in differentiating between levels 2 and 3 and levels 4 and 5 when responding to valuation tasks on the EQ-5D-5L.

A further potential issue emerging from the increased scale of use of EQ-5D and the need to adopt a more 'business-like' approach to product development is the challenge of balancing clients' needs with the long timelines associated with academic methodological research.

There have been understandable reservations within the Group about changing the descriptive system. The initial descriptive system was not developed using the techniques now available in modern psychometrics-and the Group has now embarked upon a fundamental programme of research to revisit the core descriptive system, as well as exploring the potential conceptual and empirical basis for a suite of bolt-on and bolt-off dimensions, as noted in Sect. 4.1. This research does not commit the Group to a 'new' EQ instrument-but it may ultimately lead in that direction. That decision point will be a critical one for the future of the EQ-5D: improvements in the measurement system may mean better science, but breaking away from the current EQ-5D loses the appeal to current users of history of evidence and consistency.

Notwithstanding continued objections to the use of QALYs in HTA [44], most policy responses so far have entailed more explicitly taking into account additional aspects of 'value' (such as process-of-care utility, effects 
on workforce productivity, the reduction in health inequalities) alongside the QALY (for example, the proposal to establish 'value based pricing' in the UK [45]), rather than a rejection of the QALY per se. Furthermore, while formal, QALY-based HTA processes were initially restricted to Europe, Canada, Australia and New Zealand, similar HTA processes are now being established, or actively planned, in Asian, African, Middle Eastern and South American healthcare systems [46-48]. This is likely to generate a new wave of demand for the EQ-5D.

In addition, new uses for the EQ-5D outside of clinical trials and technology appraisal-most notably, the inclusion of the EQ-5D in routine outcomes measurement in healthcare systems (i.e. PROMs programmes)—have quite considerably increased the amount of data being collected and used. A key challenge for the future will be to recognise that these new uses of the EQ-5D, which are not principally driven by cost-effectiveness, QALYs or resource allocation, address quite different questions-and that this is reflected in the development of appropriate methods and research to support these applications, as we detailed in Sect. 4.1.

Acknowledgements and disclaimers The authors are grateful to Gerben Bakker, Gouke Bonsel, Mike Herdman, Mark Oppe and Simon Pickard for their comments on earlier drafts of this paper, and to the reviewers for Applied Health Economics and Policy for their helpful comments. Funding for this article was provided by the EuroQol Research Foundation. Views expressed in the paper are the authors' and do not necessarily represent those of the EuroQol Research Foundation.

RB wrote the first draft of the historical overview, and ND wrote the first draft of the present and future material. The subsequent draft was reviewed by the five persons named in the acknowledgements. The final draft was prepared jointly by ND and RB in light of their comments and those of referees for this journal.

\section{Compliance with Ethical Standards}

Conflict of interest ND was the elected Chair of the EuroQol Group 2010-2014, and both Nancy and RB are members of the EuroQol Group.

Open Access This article is distributed under the terms of the Creative Commons Attribution-NonCommercial 4.0 International License (http://creativecommons.org/licenses/by-nc/4.0/), which permits any noncommercial use, distribution, and reproduction in any medium, provided you give appropriate credit to the original author(s) and the source, provide a link to the Creative Commons license, and indicate if changes were made.

\section{References}

1. The EuroQol Group. EuroQol—a new facility for the measurement of health-related quality of life. Health Policy. 1990;36:199-208.

2. Brooks R, on behalf of the EuroQol Group. EuroQol: the current state of play. Health Policy. 1996;37:53-72.
3. Brooks R. The EuroQol group after 25 years. Dordrecht: Springer; 2013.

4. Gudex C. The descriptive system of the EuroQol instrument. In: Kind P, Brooks R, Rabin R, editors. EQ-5D concepts and methods: a developmental history. Dordrecht: Springer; 2005. p. 19-27.

5. Feng Y, Parkin D, Devlin N. Assessing the performance of the EQ-VAS in the NHS PROMs programme. Qual Life Res. 2014. doi:10.1007/s11136-013-0537-z.

6. Dolan P, Gudex C, Kind P, Williams A. A social tariff for EuroQol: results from a UK general population survey. Discussion Paper 138. York: Centre for Health Economics; 1995.

7. Brooks R, Rabin R, de Charro F, editors. The measurement and valuation of health status using EQ-5D: a European perspective. Dordrecht: Kluwer; 2013.

8. Nice. Guide to the methods of technology appraisal. London: National Institute for Clinical Excellence; 2004.

9. Fox-Rushby J, Selai C. What concepts does the EQ-5D measure? Intentions and interpretations. In: Brooks R, Rabin R, de Charro $\mathrm{F}$, editors. The measurement and valuation of health status using EQ-5D: a European perspective. Dordrecht: Kluwer; 2003. p. $167-82$.

10. Rabin R, Herdman M, Fox-Rushby J, Badia X. Exploring the results of translating the EQ-5D into 11 European languages. In: Brooks R, Rabin R, de Charro F, editors. The measurement and valuation of health status using EQ-5D: a European perspective. Dordrecht: Kluwer; 2003. p. 191-205.

11. Rabin R, Gudex C, Selai C, Herdman M. From translation to version management: a history and review of methods for the cultural adaptation of the EuroQol five-dimensional questionnaire. Value Health. 2014;17:70-6.

12. Bonsel G, van Agt $\mathrm{H}$. The number of levels in the descriptive system. In: Busschbach J, Bonsel G, de Charro F, editors. Plenary Meeting of the EuroQol Group, Rotterdam October 1993. Rotterdam: Institute of Medical Technology Assessment; 1994. p. $115-20$.

13. Kind P, Macran S. Levelling the playing field: increasing the number of response categories in EQ-5D. In Kind P, Macran S, editors. 19th Plenary Meeting of the EuroQol Group Discussion Papers, York, UK, September 2002. York: Centre for Health Economics; 2002. p. 311-22.

14. Janssen M, Birnie E, Bonsel G. Quantification of the level descriptors for the standard EQ-5D three level system and a five level version according to 2 methods. Qual Life Res. 2008; 17:463-73.

15. Pickard S, de Leon M, Kohlmann T, Cella D, Rosenbloom S. Psychometric comparison of the standard EQ-5D to a 5 level version in cancer patients. Med Care. 2007;45:259-63.

16. Janssen M, Birnie E, Haagsma J, Bonsel G. Comparing the standard EQ-5D three level system with a five level version. Value Health. 2008;11:275-84.

17. Pickard S, Kohlmann T, Janssen M, Bonsel G, Rosenbloom S, Cella D. Evaluating equivalency between response systems: application of the Rasch model to a 3-level and 5-level EQ-5D. Med Care. 2007;45:812-9.

18. Herdman M, Kind P, Chevalier J, Gudex C, de Pourvourville G. Investigation of labels for additional EQ-5D levels: results of the main study +1 . In: Busschbach J, Rabin R, de Charro F, editors. 24th Scientific Plenary Meeting of the EuroQol Group Proceedings. The Hague: EuroQol Group; 2007. p. 99-151.

19. Lloyd A, Quadri N. Testing alternative labels for a UK English five level version of EQ-5D. In: Scalone L, Mantovani LG, editors. 25th Scientific Plenary Meeting of the EuroQol Group Proceedings. Baveno: EuroQol Group; 2008. p. 41-73.

20. Herdman M, Gudex C, Lloyd A, Janssen M, Kind P, Parkin D, Bonsel G, Badia X. Development and preliminary testing of the 
new five-level version of EQ-5D (EQ-5D-5L). Qual Life Res. 2011;20:1727-36.

21. Hennessy S, Kind P. Measuring health status in children: developing and testing a child-friendly version of EQ-5D. In: Kind P, Macran S, editors. 19th Plenary Meeting of the EuroQol Group Discussion Papers, York, UK, September 2002. York: Centre for Health Economics, 2002. p. 291-310.

22. Wille N, Badia X, Bonsel G, Burström K, Cavrini G, Devlin N, Egmar A-C, Greiner W, Gusi N, Herdman M, Jelsma J, Kind P, Scalone L, Ravens-Sieberer U. Development of the EQ-5D-Y: a child-friendly version of the EQ-5D. Qual Life Res. 2010;19:887-97.

23. Ravens-Sieberer U, Wille N, Badia X, Bonsel G, Burström K, Cavrini G, Devlin N, Egmar A-C, Gusi N, Herdman M, Jelsma J, Kind P, Olivares P, Scalone L, Greiner W. Feasibility, reliability, and validity of the EQ-5D-Y: results from a multinational study. Qual Life Res. 2010;19:887-97.

24. Szende A, Oppe M, Devlin N, editors. EQ-5D value sets: inventory, comparative review and user guide. Dordrecht: Springer; 2007.

25. van Hout B, Janssen M, Feng Y, Kohlmann T, Busschbach J, Golicki D, Lloyd A, Scalone L, Kind P, Pickard S. Interim scoring for the EQ-5D-5L: mapping the EQ-5D-5L to EQ-5D-3L value sets. Value Health. 2012;15:708-15.

26. Stolk E, Oppe M, Scalone L, Krabbe P. Discrete choice modeling for the quantification of health states: the case of the EQ-5D. Value Health. 2010;13(8):1005-13.

27. http://www.euroqol.org/eq-5d-publications/51-valuation.html.

28. Oppe M, Devlin N, van Hout B, Krabbe P, de Charro F. A program of methodological research to arrive at the new international EQ5D-5L valuation protocol. Value Health. 2014;17:445-63.

29. EuroQol Group 2016a. EuroQol Group 2015a. http://www. euroqol.org/euroqol/vision.html. Accessed 5 Feb 2016.

30. EuroQol Group 2016b. EuroQol Group 2015b. http://www. euroqol.org/euroqol/mission.html. Accessed 5 Feb 2016.

31. Appleby J, Devlin N, Parkin D. Using patient reported outcomes to improve health care. Chichester: Wiley; 2015.

32. Calvert M, Thwaites R, Kyte D, Devlin N. Putting patient-reported outcomes on the 'Big Data Road Map'. J R Soc Med. 2015;108:299-303.

33. Longworth L, Yang Y, Young T, Hernandez Alva M, Mukuria C, Rowen D, Brazier J. Use of generic and condition-specific measures of health-related quality of life in NICE decision-making: systematic review, statistical modelling and survey. Health Technology Assessment No. 18.9. 2014. http://www.ncbi.nlm. nih.gov/books/NBK261616/. Accessed 5 Feb 2016.
34. Yang Y, Rowen D, Brazier J, Tsuchiya A, Young T, Longworth L. An exploratory study to test the impact on three "bolt-on" items to the EQ-5D. Value Health. 2015;18:52-60.

35. Swinburn S, Lloyd A, Boye K, Edson-Heredia E, Bowman L, Janssen B. Development of a disease-specific version of the EQ5D-5L for use in patients suffering from psoriasis: lessons learned from a feasibility study in the UK. Value Health. 2013; 16(1156-62):36.

36. Yang Y, Brazier J, Tsuchiya A. Effect of adding a sleep dimension to the EQ-5D descriptive system: a "bolt-on" experiment. Med Decis Making. 2014;34:42-53.

37. Lorgelly P. Choice of outcome measure in economic evaluation: a potential role for the capability approach. Pharmacoeconomics. 2015;33:849-55. doi:10.1007/s40273-015-0275-x.

38. Devlin N, Lorgelly P. QALYs as a measure of value in cancer. J Cancer Policy. doi:10.1016/j.jcpo.2016-09-005.

39. Brazier J, Tsuchiya A. Improving cross-sector comparisons: going beyond the health-related QALY. Appl Econ Health Policy. 2015;13:557-65. doi:10.1007/s40258-015-0194-1.

40. Sussex J, Towse A, Devlin N. Operationalizing value-based pricing of medicines: a taxonomy of approaches. Pharmacoeconomics. 2013;31:1. doi:10.1007/s40273-012-0001-x.

41. Bansback N, Trenaman L, Bryan S, Johnson J. 2015 using routine patient reported outcome measures to enhance patient decision making: a proof of concept study. Qual Life Res. 2015;24:109.

42. Tsuchiya A, Williams A. Welfare economics and economic evaluation. In: Drummond M, McGuire A, editors. Economic evaluation in health care: merging theory with practice. Oxford: Oxford University Press; 2001. p. 27-8.

43. Brazier J, Dixon S, Ratcliffe J. The role of patient preferences in cost-effectiveness analysis. Pharmacoeconomics. 2009;27: $705-12$.

44. Kind P. Cost-effectiveness analysis: a view into the abyss. Appl Health Econ Health Policy. 2015;3:269-71.

45. Sussex J, Towse A, Devlin N. Operationalizing value-based pricing of medicines. Pharmacoeconomics. 2013;31:1-10.

46. Yong C, Jiang Y, Sun D, Duttagupta S. Evolution and influence of HTA in emerging markets. doi:10.1016/j.jval.2015.09.1805.

47. Glassman A, Giedion U, McQueston K. Priority setting for health in emerging markets. J Comp Eff Res. 2013;2:283-91. doi:10. 2217/cer.13.12.

48. Yang B. The future of health technology assessment in healthcare decision making in Asia. Pharmacoeconomics. 2009;27:891. doi:10.2165/11310280-000000000-00000. 